\title{
Melanoma cutáneo en Uruguay: características clínico-epidemiológicas y sobrevida en cohorte de pacientes de dos centros de referencia en el período 2008-2017
}

Dahiana Amarillo*, , Daniela De Boni ${ }^{\dagger}$, Florencia Nantes ${ }^{\ddagger}$, Clara Tambasco ${ }^{\ddagger}$, Carlos Brayer ${ }^{\dagger}$, Carlos Palacios ${ }^{\dagger}$, Virginia Giachero** ${ }^{\star *}$ Gabriel Krygier ${ }^{\S}$

\section{Resumen}

El melanoma cutáneo es el tumor que más ha aumentado en incidencia en los últimos años. Presenta alta letalidad en estadios avanzados. En nuestro país el promedio anual de casos sería de 129 en hombres, 117 en mujeres, con 50 y 30 muertes anuales respectivamente.

Objetivo: describir y analizar el perfil clínico-epidemiológico y sobrevida de una cohorte de pacientes portadores de melanoma cutáneo asistidos en dos centros de referencia en Montevideo en el período comprendido entre 2008 y 2017.

Método: se realizó un estudio descriptivo, retrospectivo, observacional de cohortes históricas en base a revisión de historias clínicas de los pacientes con diagnóstico de melanoma cutáneo asistidos en el Servicio de Oncología del Hospital de Clínicas y en la Unidad de Oncología cutánea del Instituto Nacional del Cáncer en el período comprendido entre 2008 y 2017, con un seguimiento de los casos hasta el 31 de julio de 2018.

Resultados: se analizaron 173 pacientes, mediana de edad 61,2 años (14-89), 50,3\% de sexo masculino y 49,7\% de sexo femenino. La forma anátomo-clínica nodular fue la más frecuente (32,7\%), seguida de la extensiva superficial $(28,9 \%)$, siendo el resto menos frecuentes. No fue alcanzada la mediana de sobrevida global (SVG), siendo la tasa de SVG a cinco años de 68,5\% y a diez años de 54,5\%. La mediana de SVG en EIV fue de 12 meses (IC 95\% 8,5-21,5).

Conclusiones: los resultados de nuestra serie en SVG son similares a los reportados a nivel mundial, al igual que en la mayoría de las características clínico-epidemiológicas. Se trata del primer trabajo con reporte de sobrevida en melanoma cutáneo en nuestro país.

Palabras clave: Melanoma

Key words: $\quad$ Melanoma

Neoplasias cutáneas

Skin neoplasms

Sobrevida

Survival

Uruguay

Uruguay

\footnotetext{
* Asistente de Servicio de Oncología Clínica, Hospital de Clínicas-INCA.

† Dermatóloga del Instituto Nacional del Cáncer.

‡ Posgrado del Servicio de Oncología Clínica.

$\S$ Profesor Agregado del Servicio de Oncología Clínica, Hospital de Clínicas.

II Asistente del Departamento Básico de Medicina.

** Profesora Agregada de Cirugía Plástica, Hospital de Clínicas.

Correspondencia: Dra. Dahiana Amarillo. Servicio de Oncología Clínica. Av. Italia esq. Las Heras. Montevideo, Uruguay.

Correo electrónico: damarillo@hc.edu.uy

Los autores declaran no tener conflictos de interés.

Este trabajo fue aprobado por el Comité de Ética del Hospital de Clínicas.

Recibido: $7 / 8 / 19$

Aprobado: 26/12/19
} 


\section{Introducción}

El melanoma es una neoplasia maligna originada a partir de los melanocitos, células responsables de la secreción del pigmento melanina. El melanoma cutáneo es por lejos el tipo de melanoma más frecuente, representando más del $90 \%$ de los casos, siendo un $5 \%$ aproximadamente melanomas oculares, menos del $2 \%$ melanomas mucosos y el resto de primitivo desconocido ${ }^{(1)}$.

Según datos de GLOBOCAN 2018, la incidencia se encuentra en aumento, estimándose un incremento de $5 \%$ para el 2020 y de más de $60 \%$ para el 2040 , donde se alcanzarán casi 470.000 diagnósticos por año ${ }^{(2)}$. Con respecto a la mortalidad, según la misma bibliografía, se espera un crecimiento de casi $75 \%$ para $2040^{(2)}$. Nuestro país no es ajeno a esta realidad, reportándose en publicaciones de la Comisión Honoraria de Lucha contra el Cáncer una tendencia creciente en la tasa de incidencia, con un porcentaje de cambio anual estimado para el período $1990-2017$ de $1,9 \%$ por año ${ }^{(3)}$. La tasa ajustada en el período 2011-2015 para el melanoma cutáneo es de 5,49 cada 100.000 habitantes. Se estima un promedio anual de 236 casos diagnosticados anualmente (129 en hombres y 117 en mujeres), con 80 muertes (50 y 30 respectivamente) $^{(3)}$.

Aunque el melanoma cutáneo comprende solo el $1 \%-4 \%$ de todos los tumores de piel, es el causante de mayor mortalidad. Es más frecuente en hombres, estimándose una relación de 2 a 1 , aunque se han reportado variaciones. Tiene una mediana de edad al diagnóstico de 45-55 años, aunque de todas maneras es de los tumores más frecuentes en jóvenes ${ }^{(4)}$.

La importancia del melanoma radica sobre todo en su alta letalidad. Si bien recientemente se han desarrollado terapias biológicas que han logrado mejorar de forma sustancial el pronóstico principalmente todo en casos de melanomas avanzados EIII y IV, no se han alcanzado las SVG logradas con los melanomas localizados. Por lo tanto, se remarca la importancia de su prevención y diagnóstico temprano, de modo que puedan ser tratados con procedimientos pretendidamente curativos.

\section{Objetivo}

Describir y analizar el perfil clínico-epidemiológico y sobrevida de una cohorte de pacientes portadores de melanoma cutáneo asistidos en el Hospital de Clínicas y en el Instituto Nacional del Cáncer de Montevideo en el período comprendido entre 2008 y 2017.

\section{Método}

Se realizó un estudio descriptivo, retrospectivo, observacional de cohortes históricas en base a revisión de historias clínicas de pacientes con diagnóstico de mela- noma asistidos en la policlínica del Servicio de Oncología del Hospital de Clínicas y en la policlínica de Oncología cutánea del Instituto Nacional del Cáncer en el período comprendido entre 2008 y 2017, con un seguimiento de los casos hasta el 31 de julio de 2018. El trabajo fue aprobado por el Comité de Ética institucional.

Se estudiaron las siguientes variables: sexo, edad, fecha de diagnóstico, características anátomo-clínicas del melanoma, presencia de ulceración y regresión, estadio al diagnóstico, síntoma de presentación, topografía del primario, tratamientos recibidos, presencia de recaída, tiempo entre el diagnóstico y la recaída (que definimos como sobrevida libre de progresión) y tiempo entre el diagnóstico y muerte por cualquier causa (que definimos como SVG).

Los datos fueron procesados y analizados con SPSS $23^{\circledR}$. Para comparar relación de variables nominales se utilizó test de chi cuadrado de Pearson y coeficiente de contingencia. Para evaluar curvas de SV se utilizó el método de Kaplan-Meyer, evaluando las diferencias con test de log-rank. Todos los resultados se consideraron estadísticamente significativos con valores de $\mathrm{p}<0,05$ (con test de dos colas).

\section{Resultados}

Se recabaron datos de los 173 pacientes tratados en las policlínicas de ambas instituciones. La edad mediana al diagnóstico fue de 61,2 años (14-89), siendo 50,3\% de sexo masculino y $49,7 \%$ de sexo femenino (tabla 1 ). El $60,7 \%$ de los pacientes presentaba fototipo I-II, $37 \%$ fototipo III. El 45,9\% presentaba antecedentes de exposición solar crónica o intermitente, con quemaduras solares en la infancia y ausencia de medidas de fotoprotección.

Ninguno de los pacientes tenía antecedente de melanoma previo.

El síntoma de consulta más frecuente fue el crecimiento/cambio de tamaño de lesión pigmentada en un $41 \%$, seguido de la aparición de un nevo $(30,4 \%)$. El $10 \%$ consultó por ulceración y $6 \%$ por síntomas de enfermedad locorregional/distancia (tabla 2).

En la tabla 3 podemos ver la topografía del melanoma cutáneo, siendo los sitios más frecuentes dorso y miembros. La topografía más frecuente en el sexo masculino fue el dorso $(29,9 \%)$, mientras que en el sexo femenino fue en miembros inferiores $(22,1 \%),\left(\chi^{2}=\right.$ $24,29, p<0,007$, coeficiente de contingencia 0,35 ).

La forma anátomo-clínica nodular fue la más frecuente $(32,7 \%)$, seguida de la extensiva superficial $(28,9 \%)$ (tabla 1). La regresión se encontró presente en $6,4 \%$ de los casos $(\mathrm{n}=11)$, mientras que la ulceración se reportó en $34,7 \%(\mathrm{n}=60)$. 


\begin{tabular}{|c|c|c|}
\hline & $N(173)$ & $\%$ \\
\hline \multicolumn{3}{|l|}{ Edad } \\
\hline Mediana & 61,4 & \\
\hline Rango & $14-89$ & \\
\hline \multicolumn{3}{|l|}{ Sexo } \\
\hline Hombres & 87 & $50,3 \%$ \\
\hline Mujeres & 86 & $49,7 \%$ \\
\hline \multicolumn{3}{|l|}{ Forma anátomo-clínica } \\
\hline Nodular & 52 & $32,7 \%$ \\
\hline Extensivo superficial & 46 & $28,9 \%$ \\
\hline Lentiginoso acral & 23 & $14,6 \%$ \\
\hline Lentigo maligno melanoma & 13 & $8,2 \%$ \\
\hline Amelánico & 8 & $5 \%$ \\
\hline Oculto & 7 & $4,4 \%$ \\
\hline Polipoideo & 5 & $3,1 \%$ \\
\hline Otros & 5 & $3,1 \%$ \\
\hline Sin datos & 15 & \\
\hline \multicolumn{3}{|l|}{ Breslow } \\
\hline IS & 14 & $8,6 \%$ \\
\hline Breslow $\leq 1$ & 34 & $22,4 \%$ \\
\hline Breslow $>1 \leq 2$ & 28 & $19,1 \%$ \\
\hline Breslow $>2 \leq 4$ & 39 & $25,7 \%$ \\
\hline Breslow > 4 & 36 & $24,3 \%$ \\
\hline Sin datos & 23 & \\
\hline Mediana Breslow & 2,1 & (Rango: 0,1-50) \\
\hline \multicolumn{3}{|l|}{ Ulceración } \\
\hline Sí & 63 & $42,3 \%$ \\
\hline No & 86 & $57,7 \%$ \\
\hline Sin datos & 10 & \\
\hline \multicolumn{3}{|l|}{ Regresión } \\
\hline Presente & 11 & $5,7 \%$ \\
\hline \multicolumn{3}{|l|}{ Estadio } \\
\hline IS & 14 & $8,4 \%$ \\
\hline I & 49 & $29,3 \%$ \\
\hline$\|$ & 56 & $33,5 \%$ \\
\hline III & 29 & $16,8 \%$ \\
\hline IV & 19 & $12 \%$ \\
\hline Sin datos & 6 & \\
\hline
\end{tabular}

Tabla 2. Síntoma de presentación de melanomas cutáneos.

\begin{tabular}{lcc}
\hline & $N$ & $\%$ \\
\hline Cambio de tamaño de nevo & 62 & 39,2 \\
Aparición de nevo & 45 & 30,4 \\
$\begin{array}{l}\text { Cambio de características de nevo } \\
\text { (ulceración, prurito, forma) }\end{array}$ & 32 & 20,2 \\
Conglomerado ganglionar & 6 & 3,8 \\
Control dermatológico & 6 & 3,8 \\
Síntomas de enfermedad a & 4 & 2,6 \\
distancia & & \\
Sin datos & 15 & \\
\hline
\end{tabular}

Tabla 3. Localización anatómica.

\begin{tabular}{lll|}
\hline & $N$ & $\%$ \\
\hline Dorso & 39 & 22,5 \\
Palmo-plantar & 30 & 17,4 \\
MMII & 25 & 14,5 \\
MMSS & 24 & 13,9 \\
Cara & 21 & 12,1 \\
Tórax & 11 & 6,4 \\
Oculto & 10 & 5,8 \\
Cuero cabelludo & 5 & 2,9 \\
Cuello & 5 & 2,9 \\
Abdomen & 3 & 1,7 \\
\hline
\end{tabular}

El $50 \%$ de los casos presentó Breslow mayor a 2 (dentro de los cuales un $25 \%$ es mayor a 4 ). Se vio una asociación estadísticamente significativa entre el aumento del espesor de Breslow y la ulceración $\left(\chi^{2}=48,5\right.$, $\mathrm{p}<0,0001$, coeficiente de contingencia 0,51$)$.

El 9,8\% (n=17) de los pacientes presentó mutación BRAF V600E, pero fue $60,1 \%$ positivo en el total de los testeados $(n=28)$.

Con respecto al estadio al debut, $8,4 \%$ fue melanoma IS; $29,3 \%$ estadio I; $33,5 \%$ estadio II; $16,8 \%$ estadio III, y $12 \%$ estadio IV.

En cuanto a los pacientes con estadios localizados, $64,1 \%(\mathrm{n}=100)$ completó tratamiento local con ampliación de márgenes y en 38,8\% $(\mathrm{n}=59)$ de los casos se realizó BGC. 


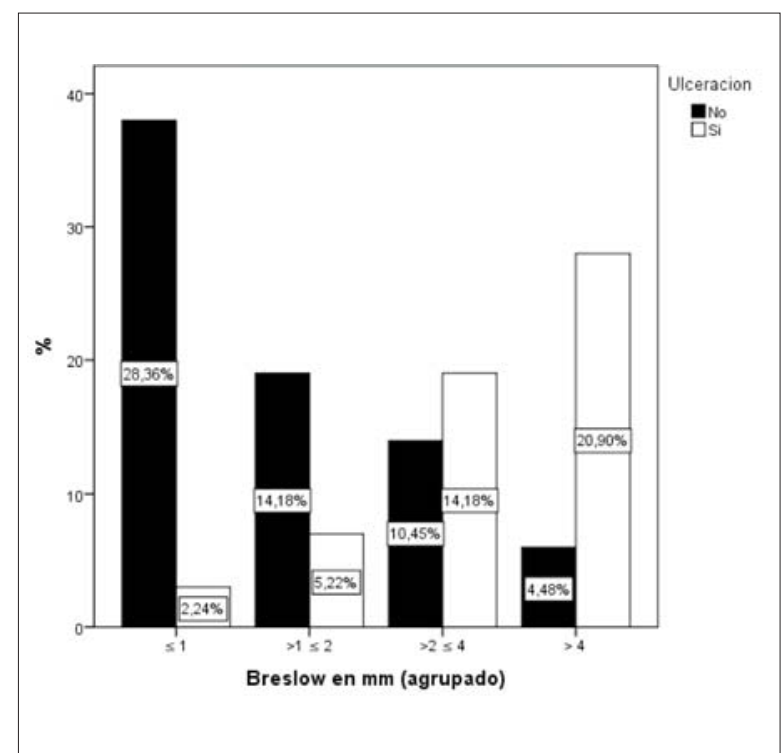

Figura 1. Relación entre ulceración y Breslow agrupado.

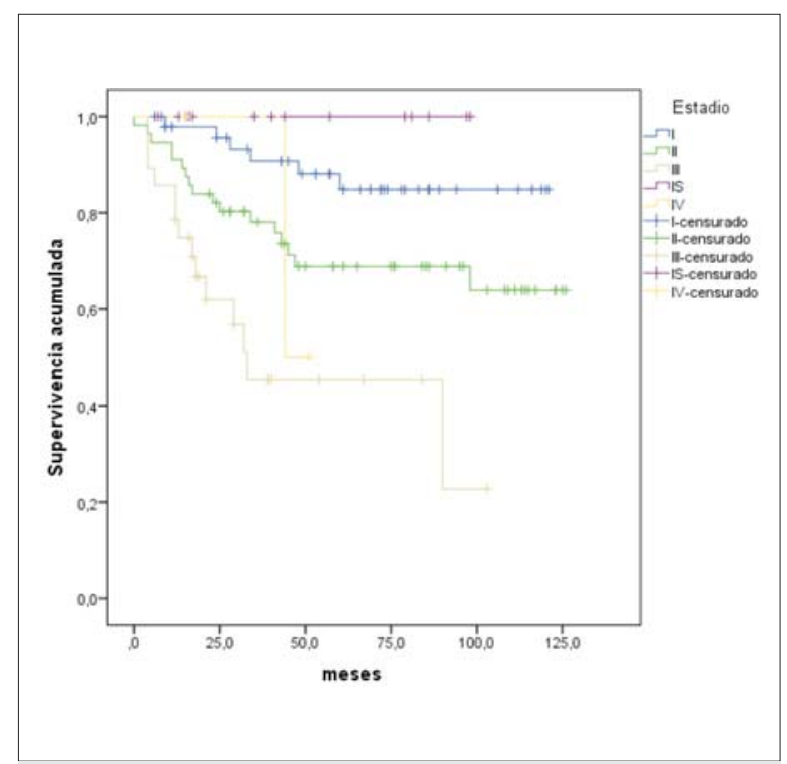

Figura 3. Sobrevida libre de enfermedad por estadio.

La BGC fue realizada en $30,4 \%$ de los pacientes con Breslow menor a $1 ; 63 \%$ entre 1 y $2 ; 63 \%$ entre 2 y 4 , y $12,9 \%$ de los pacientes con Breslow mayor a 4. De los pacientes que realizaron $\mathrm{BGC}, 7,5 \%(\mathrm{n}=13)$ presentó BGC positiva y solo en un paciente el ganglio centinela no pudo ser localizado (portador de un melanoma de dorso). De los 13 pacientes con BGC positiva, seis fueron detectados por HyE y cinco por IHQ. Nueve realizaron vaciamiento ganglionar del territorio involucrado. El resto no lo realizó, en uno de los casos por presentar

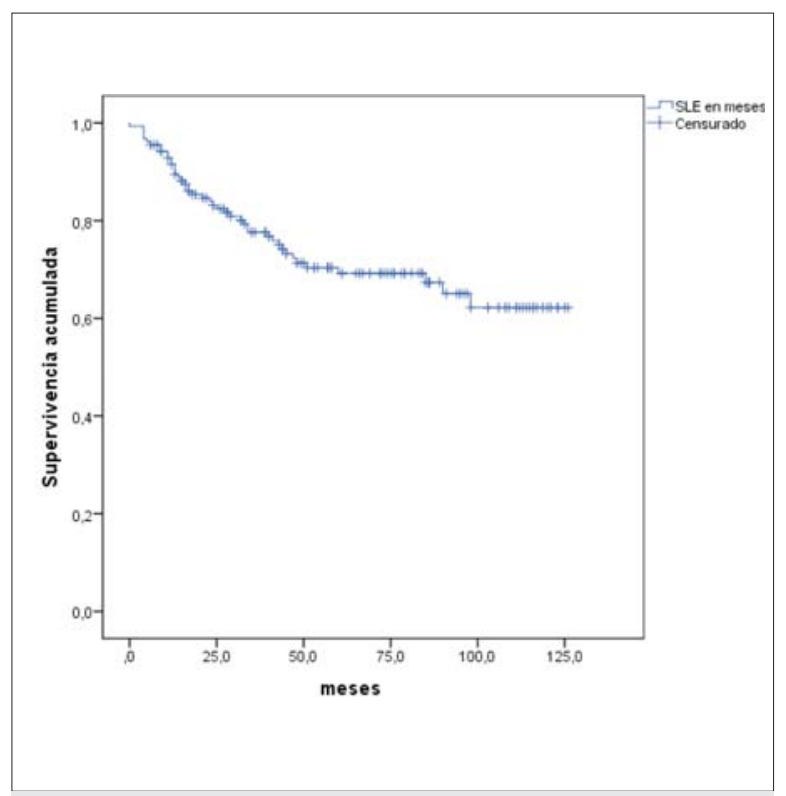

Figura 2. Sobrevida libre de enfermedad.

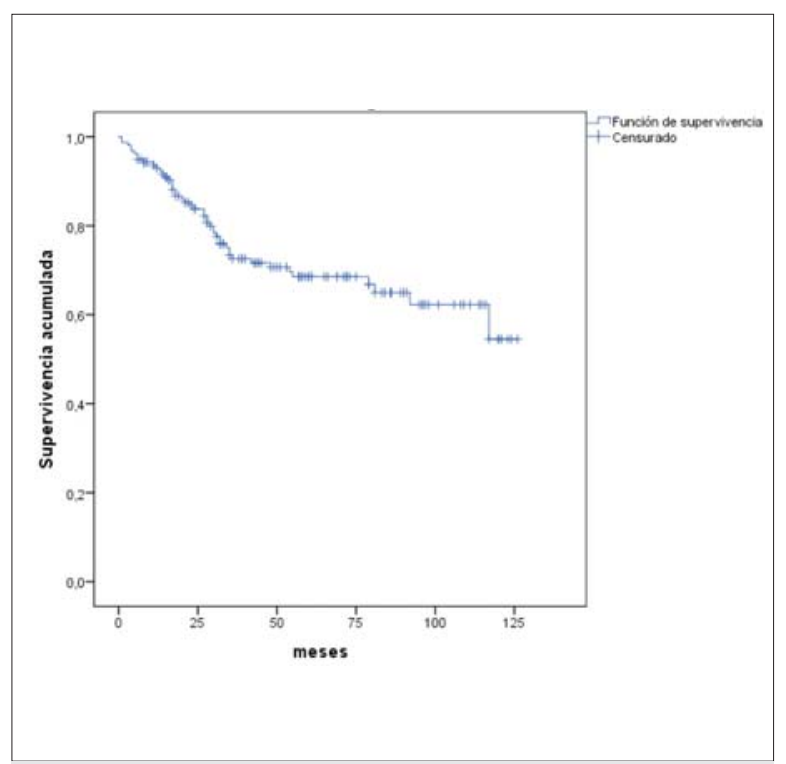

Figura 4. Sobrevida global.

solo una micrometástasis, planteándose seguimiento exclusivo, los otros tres pacientes rechazaron el vaciamiento.

El 15\% recibió tratamiento adyuvante. De los mismos, 9,8\% $(\mathrm{n}=17)$ recibió $\mathrm{RT}$ y $11 \%(\mathrm{n}=19)$ recibió IFN a dosis bajas.

La mediana de seguimiento para el total de los pacientes fue de 63 meses. La mediana de SLE no fue alcanzada, siendo la tasa de SLE a cinco años de 69,2\% (figura 2). En el estadio III la mediana de SLE fue de 33 


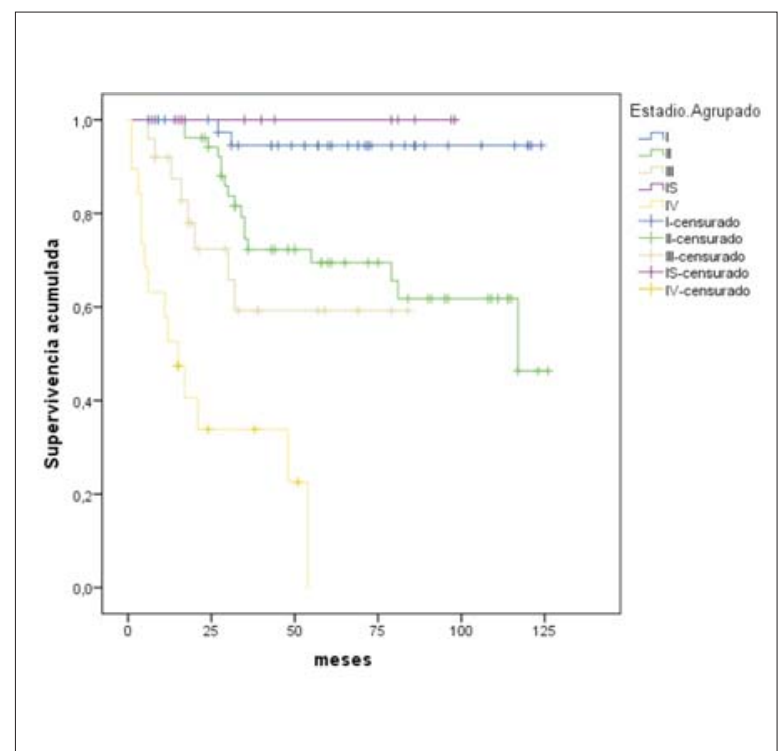

Figura 5. Sobrevida global por estadio

meses (IC 95\% 0,2-80). En los estadios I y II la mediana no fue alcanzada, con una tasa de SLE a cinco años de $63 \%$ y $84,8 \%$ respectivamente (figura 3 ). Vemos los patrones de recaída en la tabla 4.

De los 19 pacientes en estadio IV, dos recibieron tratamiento de resección completa de la enfermedad local y metastásica. Ambos recayeron, uno a los 18,5 meses y el otro a los 32 meses. El resto recibió tratamiento sistémico con criterio paliativo (siete recibieron quimioterapia, dacarbacina, carboplatino, paclitaxel), cinco IFN y un paciente inhibidores de BRAF más inhibidores de Mek, recibiendo el resto MTS.

La mediana de SVG tampoco fue alcanzada en la población total, siendo la tasa de SVG a cinco años de $68,5 \%$ y a diez años de $66,8 \%$ (figura 4 ). Si analizamos la SVG por estadio (figura 5) vemos una diferencia estadísticamente significativa ( $p<0,0001$ con test de log-rank). Solo en el EII y en el EIV se alcanzó la mediana de SVG, que fue de 117 meses (IC 95\% 49,7-184,3) y de 12 meses (IC 95\% 3,68-20,3) respectivamente.

\section{Discusión}

Este estudio es el primero en nuestro país en reportar características clínico-epidemiológicas con sobrevida en pacientes con melanoma.

La mediana de edad al diagnóstico de 61,2 años es un poco mayor que la reportada en los libros de texto ${ }^{(4)}$ que, como mencionamos, va de 45 a 55 años, aunque se indica que ésta viene en ascenso. La edad mediana suele ser menor en países desarrollados. Por ejemplo, en un estudio publicado en 2017 en base a datos de la SEER ${ }^{(5)}$, se reportó una edad mediana al diagnóstico de 54 años,
Tabla 4. Recaídas según estadio

\begin{tabular}{lcc|}
\hline & $N$ & $\%$ \\
\hline Estadio I & 4 & \\
Locorregional & 2 & $4,1 \%$ \\
A distancia & 43 & $8,2 \%$ \\
No recaída & & $87,2 \%$ \\
\hline Estadio II & 12 & \\
Locorregional & 10 & $21,2 \%$ \\
A distancia & 38 & $10,7 \%$ \\
No recaída & & $67,9 \%$ \\
\hline Estadio III & 13 & \\
Locorregional & 2 & $76,4 \%$ \\
A distancia & 13 & $46,4 \%$ \\
No recaída & & \\
\hline
\end{tabular}

siendo en un estudio alemán de similares características de 55,6 años ${ }^{(6)}$, y en un trabajo español publicado también en $2014^{(7)}$ de 57,5 años. Por otra parte, en reportes latinoamericanos encontramos medianas de edad más similares a la nuestra. Distintos trabajos muestran medianas de edad al diagnóstico de 60,1 años en Chile ${ }^{(8)}$, 60,8 en Colombia ${ }^{(9)}$ y 58,9 años en Perú ${ }^{(10)}$. En Argentina y Brasil son un poco más bajas que las nuestras y que las del resto de la región: $55-58^{(11)}$ y $58,9^{(12)}$.

En cuanto a la relación entre sexos, encontramos una discreta diferencia a favor del sexo femenino. Si bien se reporta en los libros de texto una relación 2:1 a favor del sexo masculino ${ }^{(4)}$, también se remarca que esto puede variar en las distintas poblaciones. En el reporte de la SEER también se reportó mayor frecuencia a favor del sexo masculino $-45 \%$ vs $55 \%$-, pero en el resto de los trabajos analizados la relación es variable (en Alemania, mayor cantidad de mujeres, $56 \%$ vs $43 \%{ }^{(6)}$, en Argentina $49 \%$ vs $50 \%$ a favor del sexo masculino ${ }^{(11)}$ ).

En más de un $60 \%$ de los casos se registraban fototipos bajos (1 y 2), lo cual está de acuerdo con el mayor riesgo de melanoma que presentan estos individuos. Los síntomas de presentación estuvieron vinculados a aparición o cambios de las características de lesiones pigmentadas (en casi 90\%). Vale la pena mencionar que solo 3,8\% se diagnosticó por controles dermatológicos en pacientes asintomáticos (screening), a pesar de que se trataba de individuos de fototipos claros con antecedentes de exposición solar. Esto nos hace pensar en que sería importante diseñar estrategias de prevención y screening, con énfasis en la recomendación de controles dermatológicos en sujetos de riesgo. 
Si bien muchos pacientes consultaron por cambios en un nevo previo, se reporta en la bibliografía una tasa de conversión de nevos melanocíticos en melanomas que va de $25 \%$ a $30 \%{ }^{(13-15)}$, y se plantea que la mayoría de las veces siempre se ha tratado de melanomas que han ido evolucionando.

Con respecto a la topografía, vemos que las lesiones a nivel de dorso fueron las más frecuentes $(22,5 \%)$, seguidas de las palmo-plantares $(7,4 \%)$ y las lesiones en miembros superiores e inferiores y cara $(14,5 \%, 13,9 \%$ y $12,1 \%$ respectivamente). El dorso como sitio predominante es algo que se presenta en la mayoría de los trabajos analizados, siendo generalmente las localizaciones en tronco un $30 \%-40 \%$, en extremidades $15 \%-40 \%$ y a nivel de cabeza y cuello $15 \%-40 \%^{(7,8,12,16-20)}$, similar a lo que aquí observamos. Llama la atención la frecuencia relativamente elevada a nivel de palmas y plantas en nuestra serie comparada con el resto de los estudios.

La topografía mostró una correlación significativa con el sexo $(p<0,007$, coeficiente de contingencia $0,35)$. Vemos que en el sexo masculino es más frecuente la topografía a nivel de dorso, siendo en el sexo femenino los miembros inferiores. Esto es algo que también se vio en todos los trabajos que analizamos ${ }^{(7,8,12,16-20)}$, y estaría involucrado a los patrones de exposición solar ${ }^{(21)}$.

Con respecto a las formas anátomo-clínicas, los melanomas nodulares fueron los más frecuentes. Nos llama la atención, ya que en la literatura generalmente la forma reportada como más frecuente es el melanoma en extensión superficial. Esto podría deberse a diagnósticos más tardíos, donde ya encontramos un crecimiento en fase vertical, no siempre reportándose si hay áreas de crecimiento en extensión superficial. En un trabajo español publicado en 2013 con 969 pacientes, el melanoma en extensión superficial comprendía $53,5 \%$, siendo el nodular $14,9 \%{ }^{(17)}$. En el trabajo de Ferrari y colaboradores, en Brasil ${ }^{(12)}$, la relación era de $33,8 \%$ vs $26,1 \%(\mathrm{n}=364)$, en el reporte alemán de $39,1 \%$ vs $15,3 \%(n=1.784)^{(6)}, 65,4 \%$ vs $11,1 \%$ en el trabajo español publicado por Fernández con un $\mathrm{n}=411^{(7)}$. En el reporte de 2017 con datos de la SEER ${ }^{(5)}$ con 106.511 pacientes la relación fue de $36 \%$ vs $7,1 \%$, aunque en este trabajo se vio un $45 \%$ de casos donde no se especificó el subtipo anatómico.

En cuanto al espesor de Breslow, 50\% se encuentra en el grupo de mayor a $2 \mathrm{~mm}$, los que presentan un mayor riesgo. Este parámetro es uno de los principales factores predictores del riesgo de recaída, de compromiso ganglionar y a distancia, constituyendo un factor crucial para la estadificación del T en el TNM de la AJCC en su última versión 2017. En nuestro trabajo es superior a lo que podemos observar en los países con más alto índice de desarrollo humano. En uno de los trabajos españoles se vio una mediana de Breslow de 1,61, siendo solo 22\% mayores a $2 \mathrm{~mm}^{(22)}$. En el trabajo norteamericano ${ }^{(5)}$ solo $12,3 \%$ de los pacientes tenía un espesor de Breslow mayor a 2 , siendo en $65 \%$ de los casos menor a $1 \mathrm{~mm}$. Esto va de la mano con la baja proporción de pacientes diagnosticados de forma temprana por controles dermatológicos en pacientes asintomáticos, lo que lleva a consultas más tardías y diagnóstico en estadios más avanzados. Cabe destacar que es un problema de toda la región, por ejemplo, en el trabajo de Brasil también el 50\% de los pacientes fue diagnosticado con un espesor de Breslow mayor a $2 \mathrm{~mm}^{(12,23)}$. Esto se refleja también en las diferencias que vemos en los estadios de presentación, siendo en los países más desarrollados mayor la proporción de pacientes diagnosticados en estadios más precoces en comparación con lo que vemos en nuestro estudio ${ }^{(5,17)}$.

Pocos pacientes fueron testados para BRAF, lo cual se debe a que el testeo fue incorporado en los últimos años y sigue siendo un estudio no estandarizado para los laboratorios de AP y biología molecular. El 9,8\% (n=17) de los pacientes presentó mutación BRAF V600E, siendo el $60,1 \%$ del total de los testeados (n=28). Si bien el $n$ es pequeño, está reportado en la literatura que aproximadamente el $50 \%$ de los pacientes con melanoma presentan mutaciones a nivel del gen BRAF, fundamentalmente la mutación V600 $\mathrm{E}^{(24,25)}$.

La mayoría de los pacientes que tenía indicación de tratamiento local completó el mismo, ya sea con ampliación de márgenes o BGC. Recordemos que en base al estudio MSLT1 ${ }^{(26)}$, tenemos indicación formal de BGC en aquellos pacientes con espesor de Breslow entre 1 y 4 $\mathrm{mm}$, constituyendo el grupo en el cual se vio beneficio en SV melanoma específica (tasa de SV a diez años $62 \%$ vs $85 \%$, HR 3,09, IC 95\% 2,12-4,49). En el grupo con espesor mayor a $4 \mathrm{~mm}$ la incidencia estimada de compromiso ganglionar rondaba el $44 \%$, y no mostró beneficio de la técnica ${ }^{(27)}$. En nuestro trabajo vemos que los pacientes con Breslow mayor a $4 \mathrm{~mm}$ solo en un $12 \%$ fueron sometidos a la BGC, mientras que el espesor entre 1 y $4 \mathrm{~mm}$ fue mayor a $60 \%$. En vistas a los nuevos resultados en estudios de adyuvancia, creemos que los pacientes con Breslow $\geq 4$ pueden beneficiarse de un diagnóstico precoz de su enfermedad ganglionar y un eventual tratamiento adyuvante, por lo que en nuestro centro, luego de discusión multidisciplinaria, estamos recomendando la BGC en este grupo de pacientes.

Los pacientes con estadio IV recibieron MTS o PQT en su mayoría, dado que en el tiempo en que nuestros pacientes fueron tratados no contábamos con otras opciones terapéuticas. Actualmente, si bien se tiene muy presente la indicación de immune check point inhibitors como pembrolizumab o nivolumab, e inhibidores de BRAF-MEK, solo estas últimas están cubiertas en nuestro país desde este año. 
La mediana de seguimiento para el total de los pacientes fue de 63 meses. Nuestros resultados en SLE están en relación con lo reportado en la literatura ${ }^{(4)}$. En todos los estadios las recaídas locorregionales fueron las más frecuentes. La mediana de SVG no fue alcanzada en la población total, siendo la tasa de SVG a cinco años de $68,5 \%$ y a diez años de $66,8 \%$. Si analizamos la SVG por estadio vemos una diferencia estadísticamente significativa en las curvas de SV para EI vs EII vs EIII vs EIV ( $p<0,0001$ con test de log-rank). Destacamos dentro de nuestros resultados la mediana de SVG de 12 meses en los pacientes EIV, dado que fueron tratados con PQT y MTS. Tengamos en cuenta que los ensayos con dacarbacina monodroga con criterio paliativo en pacientes EIV mostraban sobrevidas que rondaban los 6-9 meses $^{(4)}$. Se trata de un dato muy interesante, con mediana de SVG sustancialmente mayor que lo reportado en la literatura.

En el estadio I la tasa de SV a cinco años es de 94,5\%, en estadio II es de 69,5 y en el estadio III 59,2\%. En el estadio IV la tasa de SV a cinco años es de $0 \%$ y a los dos años de $33,8 \%$. Esto también está en concordancia con lo que se evidencia en la literatura ${ }^{(4)}$. Un trabajo chileno publicado en 2005 con 42 pacientes ${ }^{(8)}$, informa una SV de $100 \%$ para los EI, $70 \%$ en EII, $40 \%$ en EIII y $30 \%$ en EIV a cinco años. En un estudio suizo retrospectivo, con datos de la vida real y un total de 395 pacientes estadio IV, se vio mediana de SVG de 16,3 meses, pero cabe destacar que en dicho trabajo 121 pacientes recibieron inmunoterapia y 113 inhibidores de BRAF. En dicho estudio la mediana de SVG para los pacientes que no recibieron terapias target fue de 7,4 meses $^{(27)}$, lo cual es inferior a lo que encontramos en nuestro trabajo. Es importante remarcar que nuestro $\mathrm{n}$ es pequeño (19 pacientes), tres de los cuales fueron EIV completamente resecados, lo cual, como sabemos, mejora el pronóstico. Contamos con un trabajo prospectivo, fase II de la SWOG, que tomó 77 pacientes con melanoma metastásico cuidadosamente seleccionados, a quienes se le resecaron todas las lesiones. Con una mediana de seguimiento de cinco años, la SL de recaídas y la SVG tuvieron unas medianas de 5 y 21 meses, respectivamente ${ }^{(28)}$. Un estudio holandés publicado en 2017 con datos de la vida real en pacientes EIV, mostró una mediana de SV entre 10 y 12 meses, estando más cercano a lo encontrado en nuestro trabajo $^{(29)}$.

\section{Conclusiones}

Las características epidemiológicas y los resultados de sobrevida en esta población uruguaya están dentro de los rangos publicados mundialmente. Destacamos una mediana de SVG en pacientes estadios IV de 12 meses, siendo algo superior a la reportada en la literatura (te- niendo en cuenta que los pacientes recibieron solo quimioterapia y MTS).

Nuestros pacientes fueron diagnosticados en estadios más tardíos y con enfermedad más avanzada que en otros centros. Pensamos que sería bueno implementar y recomendar estrategias de screening para la concientización y el diagnóstico precoz.

El estudio de los aspectos clínico-patológicos del melanoma en nuestra población tiene una gran relevancia, dado que nos permite conocer resultados en la vida real, lo cual ha de contribuir a realizar los ajustes necesarios para mejorar la calidad de la práctica clínica estándar. Se trata del primer estudio epidemiológico con resultados en SVG de pacientes con melanoma cutáneo en nuestro país.

\section{Summary}

Cutaneous melanoma is the tumour whose incidence has increased the most in recent years. This condition is highly lethal in advanced stages. In our country the annual average of cases adds up to 129 in men and 117 in women, there being 50 and 30 respectively, every year.

Objective: to describe and analyse the clinical and epidemiological profile and survival in a cohort of patients who are carriers of cutaneous melanoma and were assisted in two reference centers in Montevideo between 2008 and 2017.

Methods: we conducted a descriptive, retrospective, observational study of historical cohorts, based on a review of the medical records in patients with a diagnosis of cutaneous melanoma assisted in the Oncology Service of the University Hospital and the Skin Oncology Unit of the National Cancer Institute between 2008 and 2017, with a follow-up until March 31, 2018.

Results: 173 pacientes were analysed, median age was 61.2 years old (14-89), 50.3\% were men and $49.7 \%$ were women. Nodular anatomo-clinical presentation was the most frequent form $(32.7 \%)$, followed by superficial spreading $(28.9 \%)$, other forms being less frequent. Median global survival was not achieved, global survival after 5 years being $68.5 \%$ and $54.5 \%$ after 10 years. Median global survival in Stage 4 was 12 months (IC 95\% 8.5-21.5).

Conclusions: the results in our series are similar to those reported internationally, in terms of global survival, the same as in most of the clinical an epidemiological characteristics. This is the first study with a cutaneous melanoma survival report in our country.

\section{Resumo}

O melanoma cutâneo é o tumor cuja incidência mostrou o maior incremento nos últimos anos. Nos estádios avançados apresenta alta letalidade. No Uruguai a mé- 
dia anual de casos seria de 129 em homens, 117 em mulheres, com 50 e 30 casos anuais respectivamente.

Objetivo: descrever e analisar o perfil clínico-epidemiológico e a sobrevida de uma coorte de pacientes portadores de melanoma cutâneo atendidos em dois centros de referência em Montevidéu no período 20082017.

Métodos: um estudo descritivo, retrospectivo, observacional de coortes históricas foi realizado baseado na revisão dos prontuários dos pacientes com diagnóstico de melanoma cutâneo atendidos no Serviço de Oncologia do Hospital de Clínicas e na Unidade de Oncologia cutânea do Instituto Nacional del Cáncer no período 2008-2017, com um seguimento até 31/07/2018.

Resultados: foram analisados 173 pacientes, com mediana de idade 61,2 anos (14-89), sendo $50,3 \%$ do sexo masculino e $49,7 \%$ feminino. A forma anátomo-clínica nodular foi a mais frequente $(32,7 \%)$ seguido pela extensiva superficial $(28,9 \%)$, as demais formas foram menos frequentes. Não foi possível alcançar a mediana de sobrevida global, sendo a taxa de SVG a 5 anos $68,5 \%$ e a 10 anos $54,5 \%$. A mediana de SVG em EIV foi de 12 meses (IC 95\% 8,5-21,5).

Conclusões: os resultados da série estudada em sobrevida global são similares aos descritos internacionalmente, assim como a maioria das características clínico-epidemiológicas. Este é o primeiro trabalho que informa sobre a sobrevida em melanoma cutâneo no Uruguai.

\section{Bibliografía}

1. Chang A, Karnell L, Menck H. The National Cancer Data Base report on cutaneous and noncutaneous melanoma: a summary of 84,836 cases from the past decade. The American College of Surgeons Commission on Cancer and the American Cancer Society. Cancer 1998; 83(8):1664-78.

2. International Agency for Cancer Research. Global Cancer Observatory. Lyon: IARC, 2018. Disponible en: http://gco. iarc.fr/. [Consulta: 6 octubre 2018].

3. Comisión Honoraria de Lucha Contra el Cáncer. Registro Nacional de Cáncer. Situación epidemiológica del Uruguay en relación al cáncer-Mayo 2019. Montevideo: CHLCC, 2019. Disponible en: http://www.comisioncancer.org.uy /uc_513_1.html. [Consulta: 2 noviembre 2019].

4. Ribas A, Craig L, Slingluff JrR. Cutaneous Melanoma. En: Devita V, Lawrence T, Rosenberg S. Devita, Hellman and Rosenberg's Cancer: principles \& practice of Oncology. 10 ed. Philadelphia: Wolters-Kluwer, 2015:1990-2050.

5. Enninga E, Moser J, Weaver A, Markovic S, Brewer J, Leontovich A, et al. Survival of cutaneous melanoma based on sex, age, and stage in the United States, 1992-2011. Cancer Med 2017; 6(10):2203-12.

6. Katalinic A, Kunze U, Schäfer T. Epidemiology of cutaneous melanoma and non-melanoma skin cancer in Schles-
wig-Holstein, Germany: incidence, clinical subtypes, tumour stages and localization (epidemiology of skin cancer). Br J Dermatol 2003; 149(6):1200-6.

7. Fernández I, Rivas F, Fúnez R, Bámquez N, de Troya M. Epidemiología del melanoma en una población multicultural mediterránea. Piel 2014; 29(7):401-5.

8. Iribarren O, Sepúlveda M, Hidalgo J, Madariaga J. Estudio epidemiológico de melanoma maligno en la IV Región de Chile. Cuad Cir 2005; 19(1):33-8.

9. Pozzobon F, Fierro E, Acosta Á, Carreñoc A. Características del melanoma cutáneo primario en el Instituto Nacional de Cancerología 2006-2010. Rev Colomb Cancerol 2013; 17(3):111-8.

10. Gutierrez C, Alarcón E, Valle R, Calderón G. Epidemiología del melanoma maligno en el Instituto Nacional de Enfermedades Neoplásicas , Perú , 2000-2004. Folia Dermatol Perú 2007; 18(1):23-7.

11. Loria D, González A, Latorre C. Cutaneous melanoma epidemiology in Argentina?: analysis from the Argentine Cutaneous Melanoma Registry. Dermatol Argent 2008; 14(2):436-42.

12. Ferrari N, Muller H, Ribeiro M, Maia M, Sanches J. Melanoma cutâneo: estudo epidemiológico descritivo. Sao Paulo Med J. 2008; 126(1):41-7.

13. Bevona C, Goggins W, Quinn T, Fullerton J, Tsao H. Cutaneous melanomas associated with nevi. Arch Dermatol 2003; 139(12):1620-4.

14. Damsky W, Bosenberg M. Melanocytic nevi and melanoma: unraveling a complex relationship. Oncogene 2017; 36(42):5771-92.

15. Lin W, Luo S, Muzikansky A, Lobo A, Tanabe K, Sober A, et al. Outcome of patients with de novo versus nevus-associated melanoma. J Am Acad Dermatol 2015; 72(1):54-8.

16. Duarte C, Flórez J, López H, Meneses M, de Vries E. Survival of acral lentiginous melanoma in the National Cancer Institute of Colombia. J Eur Acad Dermatol Venereol 2017; 31(3):438-442.

17. Sáenz S, Conejo-Mir J, Cayuela A. Epidemiología del melanoma en España. Actas Dermosifiliogr 2005; 96(7):411-8.

18. Ríos L, Nagore E, López J, Redondo P, Martí RM, Fernández-de-Misa R, et al. Registro nacional de melanoma cutáneo. Características del tumor en el momento del diagnóstico: 15 años de experiencia. Actas Dermosifiliogr 2013; 104(9):789-99.

19. Bellolio E, San Martín A, Quiñiñir L, Orellana J, Tapia $\mathbf{O}$, Rifo P, et al. Evaluación de factores histológicos pronósticos de sobrevida en melanoma maligno cutáneo, seguimiento de 13 años. Rev Chil Cir 2010; 62(4):331-8.

20. Thomas N, Kricker A, Waxweiler W, Dillon P, Busman K, From L, et al. Comparison of clinicopathologic features and survival of histopathologically amelanotic and pigmented melanomas: a population-based study. JAMA Dermatol 2014; 150(12):1306-314. 
21. Elwood J, Gallagher R. Body site distribution of cutaneous malignant melanoma in relationship to patterns of sun exposure. Int J Cancer 1998; 78(3):276-80.

22. Avilés-Izquierdo J, Lázaro-Ochaita $\mathbf{P}$, Suárez-Fernández R, Márquez-Rodas I, Parra-Blanco V, Escat-Cortés J. Cambios epidemiológicos en el melanoma cutáneo: estudio retrospectivo de 969 casos (1996-2010). Rev Clin Esp 2013; 213(2):81-7.

23. Schmerling R, Loria D, Cinat G, Ramos W, Cardona A, Sánchez J, et al. Cutaneous melanoma in Latin America: the need for more data. Rev Panam Salud Publica 2011; 30(5):431-8.

24. Ascierto P, Kirkwood J, Grob J, Simeone E, Grimaldi A, Maio M, et al. The role of BRAF V600 mutation in melanoma. J Transl Med 2012; 10:85.

25. Cheng L, Lopez-Beltran A, Massari F, MacLennan G, Montironi R. Molecular testing for BRAF mutations to inform melanoma treatment decisions: a move toward precision medicine. Mod Pathol 2018; 31(1):24-38
26. Morton D, Thompson J, Cochran A, Mozzillo N, Nieweg O, Roses D, et al. Final trial report of sentinel-node biopsy versus nodal observation in melanoma. N Engl J Med 2014; 370(7):599-609.

27. Mangana J, Cheng P, Kaufmann C, Amann V, Frauchiger $\mathbf{A}$, Stögner $\mathbf{V}$, et al. Multicenter, real-life experience with checkpoint inhibitors and targeted therapy agents in advanced melanoma patients in Switzerland. Melanoma Res 2017; 27(4):358-68.

28. Sosman J, Moon J, Tuthill R, Warneke J, Vetto J, Redman B, et al. A phase 2 trial of complete resection for stage IV melanoma: results of Southwest Oncology Group Clinical Trial S9430. Cancer 2011; 117(20):4740-06.

29. Jochems A, Schouwenburg M, Leeneman B, Franken M, van den Eertwegh A, Haanen J, et al. Dutch Melanoma Treatment Registry: quality assurance in the care of patients with metastatic melanoma in the Netherlands. Eur J Cancer 2017; 72:156-65.

\section{Contribución de autores}

Los autores Dahiana Amarillo, Daniela De Boni, Florencia Nantes, Clara Tambasco y Gabriel Krygier han participado en la concepción, diseño, ejecución, análisis, interpretación de los resultados, redacción y revisión crítica. El resto de los autores participó de la concepción, diseño, ejecución y revisión del trabajo.

Dahiana Amarillo, https://orcid.org/0000-0002-8615-8639, Daniela De Boni, https://orcid.org/0000-0002-0952-333X,

Florencia Nantes, https://orcid.org/0000-0002-0450-3583, Clara Tambasco, https://orcid.org/0000-0002-9771-7539,

Carlos Brayer, https://orcid.org/0000-0002-6066-4162, Carlos Palacios, https://orcid.org/0000-0003-4698-9726,

Virginia Giachero, https://orcid.org/0000-0002-4965-8634, Gabriel Krygier, https://orcid.org/0000-0002-0518-1854 